\title{
INFERRING BRAIN DEFORMATION DURING OPEN NEUROSURGERY USING CBCT ANGIOGRAPHY
}

\author{
Niels Verheyen ${ }^{1}$, David Robben $^{1}$, Daniel Ruijters ${ }^{2}$, \\ Vitor Mendes Pereira ${ }^{3,4}$, Olivier Brina ${ }^{3}$, Frederik Maes $^{1}$ and Paul Suetens ${ }^{1}$ \\ ${ }^{1}$ KU Leuven, ESAT/PSI - UZ Leuven, MIRC - iMinds, Medical IT Dept. \\ ${ }^{2}$ Philips Healthcare, The Netherlands \\ ${ }^{3}$ Neuroradiology, University Hospitals of Geneva, Switzerland \\ ${ }^{4}$ Neuroradiology, Toronto Western Hospital, University Health Network, Canada
}

\begin{abstract}
During neurosurgery the brain can deform, which is known as brain shift. The deformation can be as large as $20 \mathrm{~mm}$ and limits the accuracy of image-guided surgery.

In this work we use pre- and intra-operative CBCT angiography to infer this deformation. We first segment cortex and vessels in both images and then perform surface registration between the pre- and intra-operative segmentations. The resulting deformation field is assumed to correspond to the brain deformation and can be used to update other preoperative images. Since the intra-operative image quality is deteriorated by streak artifacts originating from the metallic stereotactic frame, our approach explores robust segmentation and registration methods that reduce the influence of these artifacts.

Our method is tested on a clinical data set of 8 patients who underwent neurosurgery. We also compare with a recently published method and rigid registration as a baseline method, showing the improved accuracy of our approach.
\end{abstract}

\section{INTRODUCTION}

During neurosurgery, the brain deforms up to $20 \mathrm{~mm}[1,2]$. This brain shift, if not corrected for, causes image-guided surgery systems to provide inaccurate pre-operative information to the surgeon. Therefore, this brain shift has to be inferred in order to update the pre-operative images such that they correspond to the intra-operative reality.

Studies [1, 2] show that deformation is not constant throughout the brain, as the magnitude is higher near the cortex, and lower for deep structures. Several factors contribute to the size and direction of the brain shift: orientation of the patient, cerebrospinal fluid (CSF) loss, tissue resection, skull opening size, etc.

Several earlier works attempt to mitigate the problems caused by brain shift. For example, Ji et al. [3] register the

David Robben is supported by a Ph.D. fellowship of the Research Foundation - Flanders (FWO). cortical surface utilizing stereo-vision. However, stereovision can only account for the deformation on the cortex, and therefore cannot calculate the brain shift in the deeper structures. Archip et al. [4] use intra-operative MRI and construct a biomechanical model of the brain. However, intra-operative MRI is not commonly available. The use of intra-operative US was investigated by Farnia et al. [5] and Reinertsen et al. [6]. They segment certain anatomical structures such as vessels and sulci in both images to drive the registration. These studies report good results, but the use of ultrasound has an intrinsic disadvantage: to obtain good image quality there needs to be physical contact between the ultrasound probe and the brain, which might require to make a larger burr hole than strictly necessary [6].

Robben et al. [7] propose to use pre- and intra-operative cone-beam computed tomography (CBCT) angiography to infer the deformation. CBCT angiography images provide good anatomical detail and can be acquired quickly at a reasonable cost. However, in practice these images suffer from streak artifacts from the metal stereotactic frame. Smit-Ockeloen et al. [8] propose a method where the CBCT angiographies are first preprocessed to reduce streak artifacts, then landmark enhanced and finally elastically registered to find the displacement field. The landmark enhancement comprises applying a vesselness filter and zeroing all voxels inside the ventricle and outside the brain segmentations. However, even after streak artifact reduction, there are remaining streak artifacts that interfere with the registration. Since their validation is limited to artificially deformed images, it is hard to predict the method's performance on real images.

In our approach, important anatomical structures in the brain, namely the cortex and vessels, are segmented and subsequently registered. Since metal streak artifacts are present in the intra-operative images, we focus on reducing their influence on the segmentation and registration. We evaluate our method on a clinical dataset of eight patients and make a quantitative comparison with the method of Smit-Ockeloen et al. [8]. 


\section{METHODS}

\subsection{Preprocessing}

We first apply the streak artifact reduction method proposed by van der Bom et al. [9]. Then, the pre- and intra-operative images are rigidly registered, using the sum of squared differences as a similarity measure. Finally, the brain is segmented using the brain extraction tool proposed by Smith [10] adapted for CBCT [8].

\subsection{Vessel segmentation}

To extract the vessels from the image, the Frangi filter [11] is used. This filter computes the eigenvectors and eigenvalues of the Hessian matrix in each voxel of the image, on multiple scales, in order to assign a vesselness measure per voxel. The vesselness measure is thresholded at a fixed value to obtain a binary segmentation. The first eigenvector, corresponding to the lowest eigenvalue, represents the direction of the vessel.

In the intra-operative image, some streak artifacts are still present, even after streak artifact reduction. Since the streaks are tubular-like structures with high intensities, they will be recognized as vessels by the Frangi filter. In order to differentiate streaks from vessels, we segment the metal objects in each slice by thresholding the intensities, and calculate the direction from each metal object's center of gravity towards each voxel in this slice and the neighboring slices. Should this direction be aligned with the first eigenvector in that voxel, then it is likely that the high vesselness value is due to a streak artifact, and we suppress this voxel. Moreover, because of the approximation that the metallic objects can be treated as point objects, the angle between the eigenvector and the direction to the metal object can differ a few degrees. Hence, we introduce a tolerance that is empirically chosen to be $10^{\circ}$.

\subsection{Registration}

Some structures will be visible in only one of the two images due to segmentation errors, streak artifacts or differences in the timing of the contrast agent injection. These structures need to be detected as outliers and ignored during the registration. Additionally, we want to exploit the sparseness of the segmentations, which might speed up registration. Therefore, we propose to register the surfaces of the segmented structures with a surface registration algorithm with outlier detection [12]. The algorithm iteratively finds corresponding points between the two surfaces and estimates the deformation field according to these correspondences. Outliers are detected on both distance and surface orientation and the nonrigid deformation field is diffusion regularized. The surfaces of the segmentations are calculated by the marching cubes algorithm. Finally, we interpolate the sparse deformation field using thin-plate splines to create a dense deformation field representing the brain shift [13].

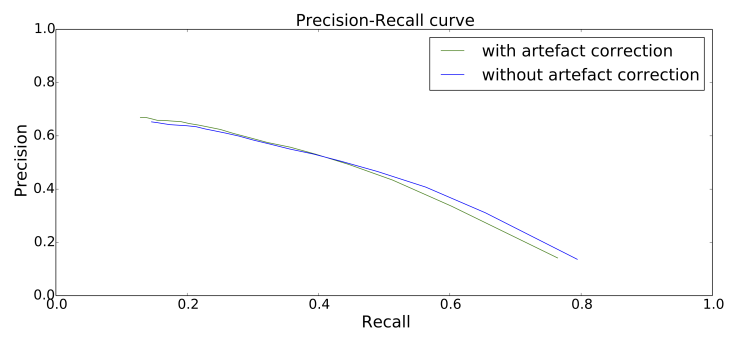

(a) Precision-recall graph of the vessel segmentation of slices without strong streak artifacts.

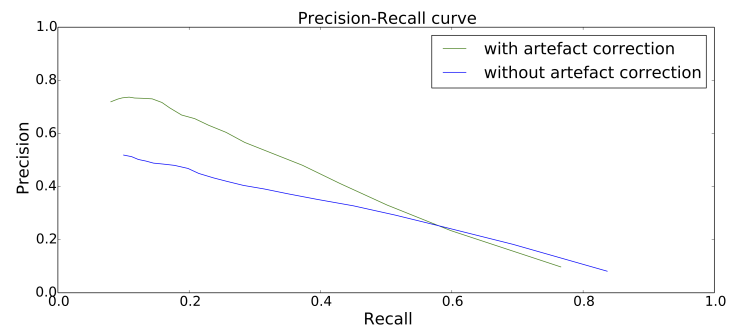

(b) Precision-recall graph of the vessel segmentation of slices with strong streak artifacts.

Fig. 1: Precision-recall graphs for both slices without many streaks and slices with many streaks. Data collected from all patients

\section{RESULTS AND VALIDATION}

All methods were tested on a data set with 8 patients undergoing neurosurgery. Images were acquired by a $\mathrm{C}$-arm CBCT machine (Philips Allura Xper FD20, in Geneva University Hospital) and have 256x256x 198 voxels with a voxel size of $0.98 \times 0.98 \times 0.98 \mathrm{~mm}$. The 8 patients all went through surgery for a clipping procedure, without any CSF drainage, except patient 1 . Patient 1 underwent a resection surgery with lumbar drainage. Mean craniotomy size was $2292 \mathrm{~mm}^{2}$.

To validate the segmentations, we manually segmented the cortex and the vasculature in each image on eight slices that are uniformly distributed over the volume.

Since the preprocessing part was adopted from [8], we only validate the more challenging intra-operative cortex segmentation here. We found that, over all images, the mean distance between manual and automatic cortex segmentation was $1.05 \mathrm{~mm}$.

\subsection{Vessel segmentation}

We subdivided the manually segmented slices into two categories: slices with and slices without strong streak artifacts. For both categories, we calculate precision-recall curves to analyze the performance of the Frangi filter and the Frangi filter with artifact correction. Fig. 1a shows that, when no streaks are present, both methods have about the same precision and recall. Fig. 1b shows that, when streaks are present, 


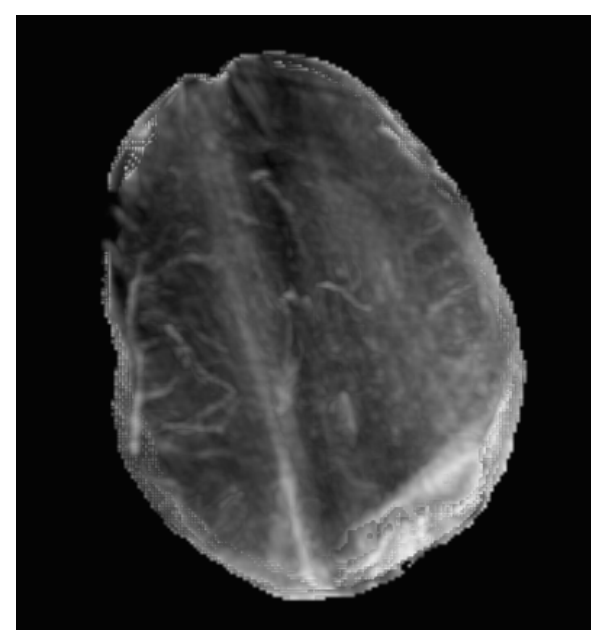

(a) Maximum intensity projection.

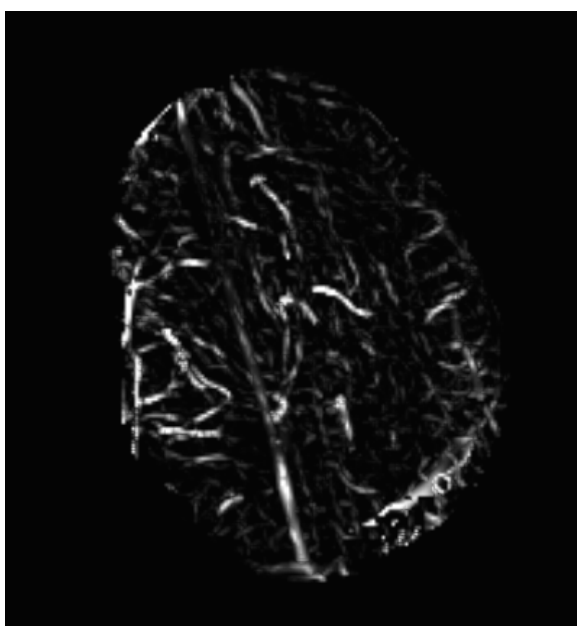

(b) Frangi filter without artifact suppression.

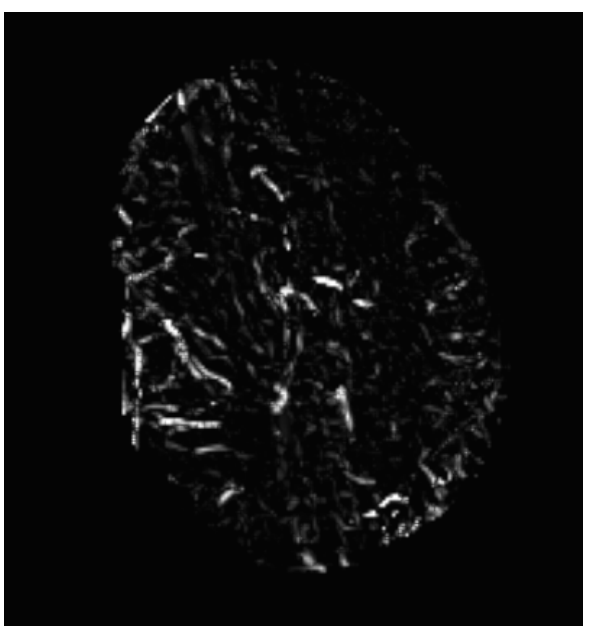

(c) Result after artifact suppression.

Fig. 2: Demonstration of the Frangi filter with and without the proposed streak suppression method on a slab of slices of the intra-operative $\mathrm{CBCT}$ angiography scan of patient 3 .

the artifact correction greatly improves the precision. Only at high recall rates, the precision is lower. This is because the artifact reduction will inherently delete some true vessels, and hence to achieve a very high recall, it will need a lower vesselness threshold which results in more false positives.

Fig. 2 visualizes the difference between the Frangi filter with and without artifact correction, where one can clearly see the streak artifact being removed from the output. However, some vessels are deleted as well.

\subsection{Registration}

The accuracy of the registration was measured by manually annotating 3D landmarks on the pre- and intra-operative images, and after registration, calculating the Euclidean distances between these corresponding points (the registration error). Eight landmarks were used for each patient ( 2 ventricle landmarks, and 6 vessel bifurcations). The mean registration error for the baseline method - rigid registration equals $4.31 \mathrm{~mm}$. The proposed method with artifact correction achieves a mean registration error of $3.12 \mathrm{~mm}$, which is an improvement over current clinical practice. However, the registration error for the proposed method without artifact correction is only $2.80 \mathrm{~mm}$. Apparently, the outlier detection of the registration framework successfully ignores the streak artifacts, making the streak correction in the vessel segmentation not strictly necessary, while the few true vessels that are accidentally removed, are informative for finding the correct transformation. We also validated the method of Smit-Ockeloen et al. [8], which has a mean registration error of $2.89 \mathrm{~mm}$ - slightly worse than ours. A more detailed look at the results (Fig. 4), shows our method gives the best result on seven out of eight images. Only in patient 4 , whose scan was affected the most by streaks, the registration failed.
The median improvement of the mean registration error is $0.71 \mathrm{~mm}$. Visually (figure 3), the registration framework captures the brain shift, even though the segmented structures are sparse. However, a collapse of the ventricles can not be accounted for, since little vasculature is present near the ventricles.

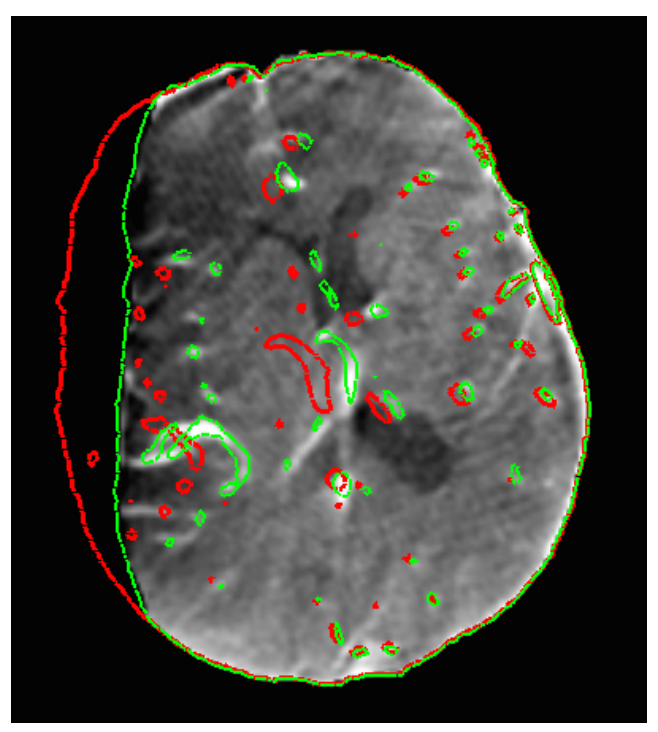

Fig. 3: Visualization of the registration of patient 1. The red contour is the pre-operative segmentation before registration and the green contour is after registration. The contours are overlaid on the intra-operative grey-scale image. 


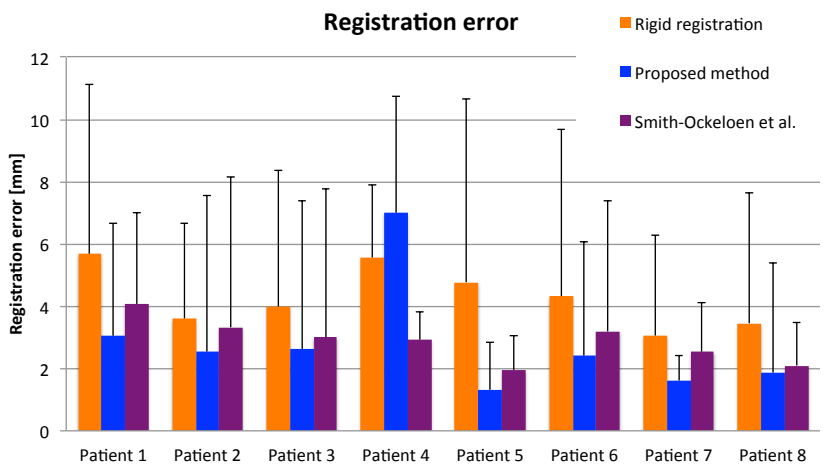

Fig. 4: Mean and maximum registration error in $\mathrm{mm}$ for different methods.

\section{CONCLUSION}

We proposed a method to register pre- and intra-operative CBCT angiographies. We showed it outperforms a state-ofthe-art method and is an improvement over the current medical practice.

In future work, we will first address the root cause of the problems: the metal streak artifacts. If the metal stereotactic frame could be replaced by a synthetic non-metal frame, the streak artifacts would disappear, and a better segmentation and registration could be achieved. Second, we will segment the ventricles and use them as an extra anatomical structure during registration. The ventricles are visible on CBCT angiography and it has been shown that they deform during surgery [1]. Since little vasculature is present near the ventricles, we expect that this additional information would improve the the registration.

\section{REFERENCES}

[1] T. Hartkens, D.L.G. Hill, A.D. Castellano-Smith, D.J. Hawkes, C.R. Maurer, Martin A.J., W.A. Hall, H. Liu, and C.L. Truwit, "Measurement and analysis of brain deformation during neurosurgery," IEEE Transactions on Medical Imaging, vol. 22, pp. 82-92, 2003.

[2] M.E. Ivan, J. Yarlagadda, A.P. Saxena, A.J. Martin, P.A. Starr, W. Sootsman, and P.S. Larson, "Brain shift during bur hole-based procedures using interventional MRI," $J$ Neurosurg, vol. 121, pp. 149-160, 2014.

[3] S. Ji, X. Fan, D.W. Roberts, A. Hartov, and K.D. Paulsen, "Cortical surface shift estimation using stereovision and optical flow motion tracking via projection image registration," Medical Image Analysis, vol. 18, pp. 1169-1183, 2014.

[4] N Archip, O. Clatz, S. Whalen, D. Kacher, A. Fedorov, A. Kot, N. Chrisochoides, F. Jolesz, A. Golby,
P.M. Black, and S.K. Warfield, "Non-rigid alignment of pre-operative MRI, fMRI, and DT-MRI with intraoperative MRI for enhanced visualization and navigation in image-guided neurosurgery," NeuroImage, vol. 35, pp. 609-624, 2007.

[5] P. Farnia, A. Ahmadian, T. Shabanian, N. D. Serej, and J. Alizeraie, "Brain-shift compensation by non-rigid registration of intra-operative ultrasound images with preoperative MR images based on residual complexity," Int J CARS, 2014.

[6] I. Reinertsen, F. Lindseth, G. Unsgaard, and D.L. Collins, "Clinical validation of vessel-based registration for correction of brain-shift," Medical Image Analysis, vol. 11, pp. 673-684, 2007.

[7] D. Robben, D. Smeets, D. Ruijters, M. Hoffmann, L. Antanas, F. Maes, and P. Suetens, "Intra-patient nonrigid registration of 3D vascular cerebral images," in MICCAI CLIP workshop, Nice, France, 2013, vol. 7761 of LNCS, pp. 106-113.

[8] I. Smit-Ockeloen, D. Ruijters, M. Breeuwer, D. Babic, O. Brina, and V.M. Pereira, "Accuracy assessment of CBCT-based volumetric brain shift field," in MICCAI CLIP workshop, Munich, Germany, 2015, vol. 9401 of LNCS.

[9] I.M.J. van der Bom, S.Y. Hou, A.S. Puri, G. Spilberg, D. Ruijters, P. van de Haar, B. Carelsen, S. Vedantham, M.J. Gounis, and A.K. Wakhloo, "Reduction of coil mass artifacts in high-resolution flat detector conebeam CT of cerebral stent-assisted coiling," Am J Neuroradiol, vol. 34, pp. 2163-2170, 2013.

[10] S.M. Smith, "Fast robust automated brain extraction," Human Brain Mapping, vol. 17, pp. 143-155, 2002.

[11] A Frangi, W Niessen, and K Vincken, "Multiscale vessel enhancement filtering," in MICCAI, William M. Wells, Alan Colchester, and Scott Delp, Eds., Cambridge, MA, 1998, vol. 1496 of LNCS, pp. 130-137, Springer Berlin Heidelberg.

[12] J. Snyders, D. Vandermeulen, P. Suetens, and P. Claes, "Non-rigid surface registration algorithms: Technical details and comparison," Tech. Rep. KUL/ESAT/PSI/1404, ESAT, KU Leuven, 2014.

[13] S. Klein, M. Staring, K. Murphy, M.A. Viergever, and J.P.W. Pluim, "Elastix: a toolbox for intensity-based medical image registration," IEEE Transactions on Medical Imaging, vol. 29, no. 1, pp. 196-205, 2010. 